\title{
Porous Bulk Metallic Glass Fabricated by Powder Consolidation
}

\author{
Z.Y. Suo, S. W. Liu, L. Zhang, H. L. Gao, H. Y. Zhang and K. Q. Qiu*
}

School of Materials Science and Engineering, Shenyang University of Technology 110023, CHINA

*For Correspondence: Dr. Ke-Qiang Qiu, 58 South Xinghua Street, Shenyang University of Technology, PIN-110023, Shenyang, CHINA, Email: kqqiu@sut.edu.cn

\section{ABSTRACT}

A synthesis method for the production of porous bulk metallic glass (BMG) is introduced. This method utilizes the superplastic forming ability of amorphous powder in the supercooled liquid (SCL) state and intenerating salt mixture as a placeholder to produce BMG foam by using a hot die pressing method. Scanning electron microscope (SEM), x-ray diffraction (XRD) and differential scanning calorimetry (DSC) were employed to characterize the morphologies of foaming structure, the crystallization and percentage of amorphous phase of the as-produced porous BMG. The results suggest that the formation of porous structure by superplastic forming process is feasible. Good bonding effect was observed between amorphous powder particles. None of crystalline phases was formed during hot pressing, and less than $3.5 \%$ percent of residual salt was enclosed in the foam. In order to remove any residual salt particles, salt preform with three-dimensional network and good connectivity is necessary.

Keywords: porous BMG; powder consolidation; hot pressing; placeholders; superplastic forming

\section{INTRODUCTION}

Since porous BMGs exhibited ductility and biologic compatibility[1], many researchers were focused on the fabrication of such excellent materials. Schroers et al [2] demonstrated the first porous BMG by entrapping gas in a liquid $\mathrm{Pd}_{43} \mathrm{Ni}_{10} \mathrm{Cu}_{27} \mathrm{P}_{20}$ alloy. Wada and Inoue [3] reported foaming of the same alloy by casting around soluble $\mathrm{NaCl}$ placeholders. Shortly afterwards, Brothers and Dunand [4] developed an alternative method based on liquid filtration of beds of hollow carbon microspheres, appropriate for use with reactive commercial BMG alloys, e.g. Zr-based alloys. In his another report, a 
method based on a salt replication process for the processing of open celled foams from the commercial BMG alloy Vit106 $\left(\mathrm{Zr}_{57} \mathrm{Nb}_{5} \mathrm{Cu}_{15.4} \mathrm{Ni}_{12.6} \mathrm{Al}_{10}\right)$ was also described [5]. Recently, Ren et al [6] fabricated a porous BMG of $8 \mathrm{~mm}$ in diameter, which is the largest porous BMG reported up to now.

Until now, Bulk amorphous foam generally fabricated by liquid route, which arises many problems. Two important problems of these are the reduction of glass forming ability (GFA) of based alloy and the contamination of liquid alloy during processing. Reports offer evidence that amorphous metal powders can be consolidated in the SCL region with little or no loss of strength by using hot pressing [7], warm rolling [8], and conventional area reduction [9,10] or equalchannel angular [11] warm extrusion techniques. It is easy to envision an incorporation of placeholders into these consolidated compacts, providing a natural method for making low-density porous materials with low processing temperatures. Recently, Qiu et al [12] fabricated bulk metallic glass foam by powder hot pressing. Less than $6.5 \%$ percent of crystalline phases were formed after hot pressing 15 to 18 minutes. In this paper, we introduce a SCL-state foaming route by blending placeholders, usually salts with controllable intenerating temperature, with amorphous metal powder to produce a porous BMG. But the powder consolidation by hot pressing is limited in 10 minutes.

\section{EXPERIMENTAL}

A pre-alloying ingot with a nominal composition of $\mathrm{Zr}_{55} \mathrm{Cu}_{30} \mathrm{Al}_{10} \mathrm{Ni}_{5}$ was prepared by induction melting the mixture of $\mathrm{Zr}, \mathrm{Cu}, \mathrm{Al}$ and $\mathrm{Ni}$ with a purity of $99.5 \%$ or better in a $\mathrm{BN}$ crucible at high vacuum. The $\mathrm{Zr}_{55} \mathrm{Cu}_{30} \mathrm{Al}_{10} \mathrm{Ni}_{5}$ powders were produced from the pre-alloying ingots by high pressure Ar gas atomization at a dynamic pressure of $3.5 \mathrm{MPa}$ after heating to $1623 \mathrm{~K}$ using an annular nozzle having a melt delivery inner diameter of $2.7 \mathrm{~mm}$. Following atomization, powders were screened to size ranges of powder particles having diameters not larger than $25 \mu \mathrm{m}$. The particle size of the metal powder is not critical but the metal particles must be considerably smaller than salt particles that are chosen as the placeholder [13].

During SCL region processing, heating into the SCL region would enhance the kinetics of crystallization. Consequently the time allowed for processing would be limited. Therefore, the processing temperature for the hot pressing has to be carefully determined by considering the time scale characterizing plastic forming is shorter than that characterizing crystallization. To determine the appropriate hot press time available before crystallization at a certain temperature, a time-temperature-transformation (TTT) plot was constructed for the amorphous powder, as shown in Fig.1, by performing isothermal DSC scans. This was accomplished by first heating the powder to selected 
annealing temperatures above $T_{\mathrm{g}}$ and then measuring the time until the sample starts to crystallize, which is indicated by the onset of an exothermic reaction.

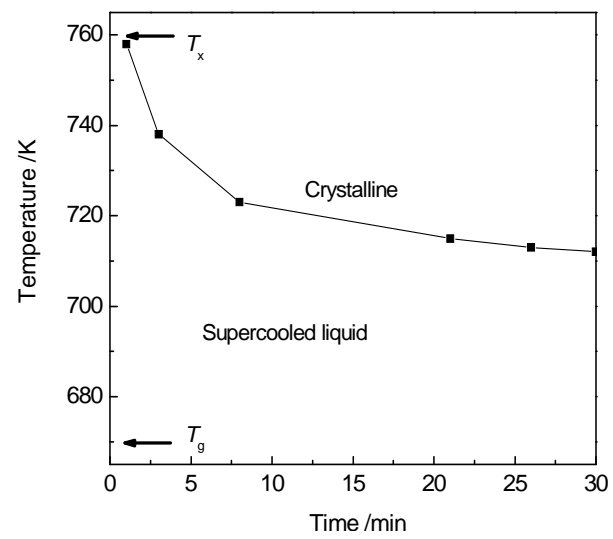

Figure 1. Time-Temperature-Transformation diagram for the onset of crystallization of gas atomized powder isothermal heated to selected temperature.

A mixture of $\mathrm{NaCl}$ and $\mathrm{CoCl}_{2}\left(\mathrm{NaCl}: \mathrm{CoCl}_{2}=7: 10\right.$ in weight) was first melted at $873 \mathrm{~K}$ for $1 \mathrm{~h}$ in low vacuum and then cracked and screened to average size of $300 \mu \mathrm{m}$. The placeholder was prepared from such a mixture with a relatively low melting temperature. The measuring intenerating temperature of the mixing salt is 643K. The particle size of the salt powder selected will be according to the intended cell size of the final foam.

The amorphous powder and salt powders were mixed with a volume fraction of amorphous powder 0.3 of all solid materials excluding pores in the mixture. Ethanol was used as the binder and a small amount, roughly 1vol.\% of the amorphous powder/salt mixture, was added during mixing. The amorphous powder/salt powder mixture was poured into a copper can and both ends of the can were vacuum sealed after outgassed. The dimension of the copper can is $30 \mathrm{~mm}$ deep by $20 \mathrm{~mm}$ inner diameter cavity and 23 mm outer diameter.

The experiment was performed using hot die pressing for consolidation. The can was placed into a hot die which was isothermal heated at $713 \mathrm{~K}$. Once the copper can was placed into the hot die the hot pressing at a constant ram pressure of $800 \mathrm{MPa}$ through a die was applied. The hot pressing time was set as $5 \mathrm{~min}$ and $10 \mathrm{~min}$ respectively. After pressing, the copper can was first quenched into water with ambient temperature, and 
then removed the copper coat. The salt was removed by flowing water, leaving the resultant foam.

The structure of the as-produced foam samples was examined by an S-3400 scanning electron microscope (SEM). X-ray diffraction (XRD) analyses and weight measurements were also carried out to determine the extent of amorphous structure and salt removal.

\section{RESULTS AND DISCUSSION}

The typical cellular structure of the as-produced foams is shown in Fig.2. Fig.2(a) shows a SEM macrograph of the foam. The foam exhibits uniformly distributed open cells and a network of well-bonded metal particles. It has a porosity of $70 \%$ and cell size in the range of 250-450 $\mu \mathrm{m}$. Fig.2(b) shows the cellular structure of the as-produced foam. The powder particles are well connected and some tiny holes among the connected particles are observed. Fig.2(c) shows the wall structure in a typical cell. The bonding region between particles as indicated by arrows indicates that strong bonding has formed between the particles. Many tiny cross lines were observed on the surface of the particles, showing that the particles have suffered severe deformation during hot pressing.

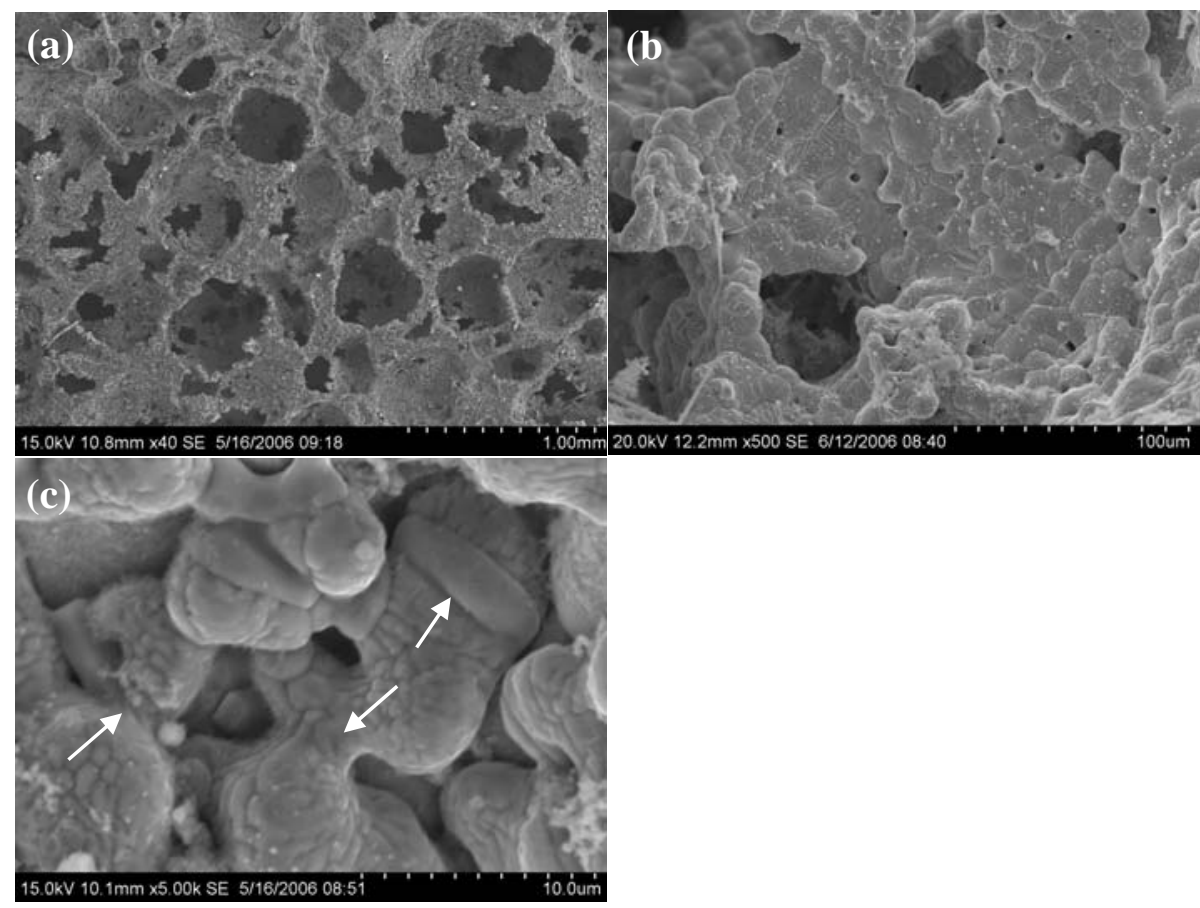

FIG. 2 (a) Morphology of as-produced foam synthesized by hot pressing. (b) The cellular structure of the as-produced foam. (c) Magnified view of the inner wall of the foam, the arrows indicating the formation of bonding areas during hot pressing. 
XRD was adopted to analyze the amorphous structure of the as-produced foam, as shown in Fig.3. It is obvious that the two as-produced foams are almost in amorphous state with a broad diffraction. DSC scans were performed on the powder metallic glass and BMG foam (produced by hot pressing for about $10 \mathrm{~min}$ ) as shown in Fig.4. Both of the amorphous powder and BMG foam show distinct glass transition temperature and SCL region, and there is no obvious difference between those characteristic temperatures. The glass transition temperature, $T_{\mathrm{g}}$, crystallization temperature, $T_{\mathrm{x}}$, the temperature interval between $T_{\mathrm{g}}$ and $T_{\mathrm{x}}$, i.e. SCL region $\Delta T_{\mathrm{x}}$, and crystallization enthalpies $\Delta H$ are in Tab1. It is that crystallization enthalpies between the two materials are almost same. So the percentage of amorphous phase can be estimated from the relatively same enthalpies in the BMG foam. Such estimation is coincident with the XRD results.

In most cases, the salt particles in the hot pressing preforms could not be dissolved completely. Some salt particles remained in the resultant foam. The residual salt can be estimated according to the expression suggested by Zhao et al [13]. For each specimen, the weights of the initial amorphous powder, the salt powder, a sample cut from the hot pressing preform, and the resultant amorphous foam were measured using a balance to an accuracy of $0.001 \mathrm{~g}$. The fraction of the residual salt in the amorphous foam with respect to the total salt in the initial hot pressing preform, $\varphi$, was determined by [12]:

$$
\varphi=\frac{W_{f}-W_{p} f_{\text {amor }}}{W_{p}\left(1-f_{\text {amor }}\right)}
$$

Where $W_{f}$ and $W_{p}$ are the weights of the amorphous foam and the corresponding predissolution hot pressing preform respectively, and $f_{a m o r}$ is the amorphous powder weight fraction in the initial mixture. The calculation result shows that the fraction of the residual salt powder in the foam with respect to the total salt in the preform is less than $3.5 \%$. The relative density of the foam is therefore expected to be a little higher than the value estimated from the initial amorphous powder fraction. With a high volume fraction of salt in the preform, most salt particles are in contact with each other and form a continuous three-dimensional network. Because all the salt particles in the network can be dissolved away by water, there is only small amount of residual salt particles in the resultant foam. In contrast, with a low volume fraction of salt in the preform some salt particles are enclosed completely by the amorphous matrix. Those isolated salt particles cannot be dissolved away and remained in the foam. Therefore hot pressing the mixture of salt and amorphous powder is only suitable to those foams with high porosity and open cell. In order to fabricate adjustable porosity, a salt perform with three-dimensional network and a required porosity is necessary. This will be reported elsewhere. 


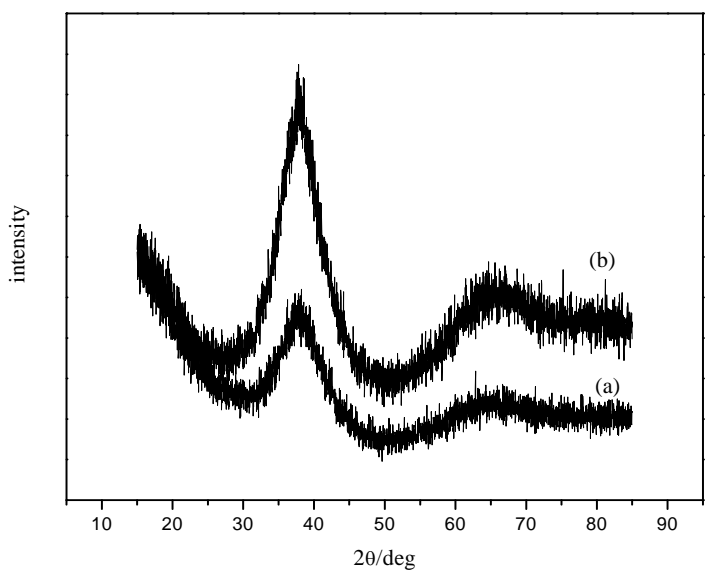

Figure 3. XRD patterns from as-produced foams synthesized by hot pressing for (a) 5 min and (b) 10 min respectively

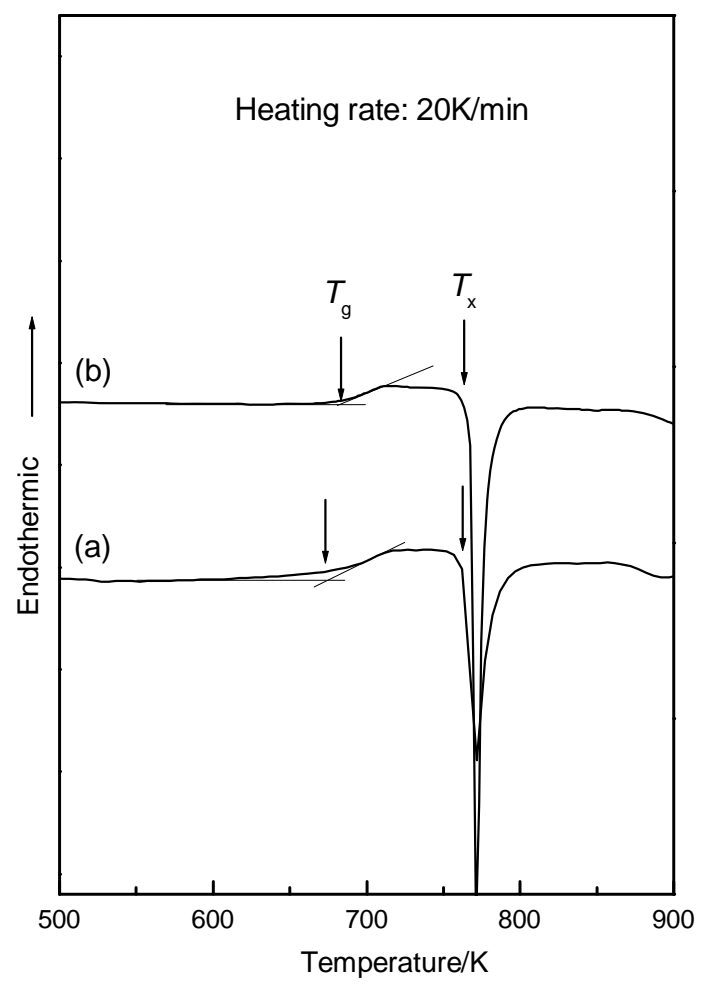

Figure 4. DSC curves of as-cast BMG with a diameter of $3 \mathrm{~mm}$ (a) and as-produced foam (b). 
The amount of tiny holes in the cell wall or struts is determined by the particles morphology and size of the amorphous and salt powder as well as the compacting pressure. It is from Fig.2 (b) and (c) that there exists some tiny holes, less than $3 \mu \mathrm{m}$ in diameter. Under the current compacting conditions, the tiny holes might reduce the strength and increase the porosity of BMG foam. Considering the enclosed residual salt existed in the foam that has reduced the porosity of the foam, the porosity of the foam is somewhat unaffected.

The large resistance of $\mathrm{Zr}_{55} \mathrm{Cu}_{30} \mathrm{Al}_{10} \mathrm{Ni}_{5}$ BMG to crystallization results in a SCL region that is accessible on a convenient time scale for processing, as shown in Fig.1. This allows for a superplastic foaming (SPF) process, where the amorphous powder behaves like a plastic. An ideal amorphous powder for SPF would be identified by a large supercooled liquid region, no embrittlement during heating treatment. An amorphous powder with a low $T_{\mathrm{g}}$ is desirable due to it reduces heating temperature and contamination come from oxidation. The SCL region of BMG foam shows smaller than that of the amorphous powder, as shown in Tab1. The glass forming ability of BMG foam is reduced in some sort. Though the SCL region of BMG foam is small, the thermal stability is increased because of its $T_{\mathrm{g}}$ is increased.

Table 1. Glass transition temperature $T_{\mathrm{g}}$, crystallization temperature $T_{\mathrm{x}}$, supercooled liquid region $\Delta T_{\mathrm{X}}$ and crystallization enthalpies $\Delta H$ of the amorphous powder and BMG foam at heating rate of $20 \mathrm{~K} / \mathrm{min}$.

\begin{tabular}{ccccc}
\hline sample & $T_{\mathrm{g}}(\mathrm{K})$ & $T_{\mathrm{x}}(\mathrm{K})$ & $\Delta T_{\mathrm{x}}(\mathrm{K})$ & $\Delta H(\mathrm{~J} / \mathrm{g})$ \\
\hline (a) powder & 672 & 762 & 90 & 52.06 \\
(b) foam & 682 & 764 & 82 & 51.70 \\
\hline
\end{tabular}

It should be noticed that when the designed mixture of salt powder is heated in the SCL-state of amorphous powder, the mixing salt intenerates and will not be as obstacles but as transfers of pressure during pressing, because the salt liquid is incompressible.

\section{CONCLUSIONS}

A new process, SPF, has been developed for manufacturing BMG foam. In SPF, amorphous powder and salt powder are first mixed at specified ratio, outgassed and sealed in a copper can. The can is then isothermal heated and compacted into a preform. During hot pressing, the amorphous powder is deformed plastically in the SCL region. A BMG foam can be successfully synthesized by this method. The particles among amorphous powder were well bonded. XRD and DSC results show that crystalline phases 
were not formed during hot pressing, and BMG foam was absolutely made of amorphous phase. Less than 3.5\% percent of residual salt was enclosed in the foam. In order to remove any residual salt particles, salt preform with three-dimensional network and good connectivity is necessary.

\section{REFERENCES}

[1] Brothers, A. H. and Dunand, D. C., 2006, Script Mater. J., vol. 54, pp. 513.

[2] Schroers, J., Veszey, C. and Johnson, W. L., 2003, Appl Phy Lett. J., vol. 82, pp. 370.

[3] Wada, T. and Inoue, A., 2003, Mater Trans JIM. J., vol. 44, pp. 2228.

[4] Brothers, A. H. and Dunand, D. C., 2004, Appl Phy Lett. J., vol. 84, pp.1108.

[5] Brothers, A. H., Scheunemann, R., DeFouw, J. D. and Dunand, D. C., 2005, Script Mater. J., vol. 52, pp. 336.

[6] Ren, Y. L., Suo, Z. Y. and Qiu, K. Q., 2006, Acta Metal Sina. J., vol. 42, pp. 379.

[7] Lee, P. Y., Hung, S. S. and Hsieh, J. T., 2002, Intermetal. J., vol. 10, pp. 1277.

[8] Kim, H. J., Lee, J. K. and Shin, S. Y., 2004, Intermetal. J., vol. 12, pp. 1109.

[9] Kawamura, Y., Kato, H., Inoue, A. and Masumoto, T., 1995, Appl Phy Lett. J., vol. 67, pp. 2008.

[10] Kato, H., Kawamura, Y., Inoue, A. and Masumoto, T., 1997, Mater Sci Eng A. J., vol. 226, pp. 458.

[11] Robertson, J. J., Im, T. and Karaman, I., 2003, Non-Crystal Solid. J., vol. 317, pp. 144.

[12] Qiu, K. Q. and Ren, Y. L., 2006, Beijing Inernational Material Forum.

[13] Zhao, Y. Y. and Sun, D. X., 2001, Script Mater. J., vol. 44, pp. 105. 